\title{
Motivos de Ingresso nos Programas de Exercícios Físicos Oferecidos pelo Serviço Social do Comércio - SESC-DF
}

\author{
Mônica da Silva Castro* \\ Márcia Miranda* \\ Nádia Lima da Silva** \\ Alexandre Palma ${ }^{* * * *}$ \\ Helder Guerra de Resende ${ }^{* * * * *}$
}

\begin{abstract}
Resumo: O objetivo deste estudo foi identificar os motivos de ingresso dos praticantes de exercícios físicos dos Centros de Atividades do SESC-DF e verificar se eles se diferenciam conforme sexo, idade e tipos de exercícios físicos. Uma amostra representativa de 986 indivíduos de ambos os sexos (com 15 anos ou mais) respondeu um questionário construído e validado por Alves et al. (2007), cujos dados revelam que os sujeitos ingressaram nos programas (a) por indicação médica, (b) para evitar problemas de saúde e (c) para melhorar o condicionamento físico. Esses resultados sugerem que o argumento da saúde é o principal motivo que justifica a adesão aos programas de exercícios físicos do SESC-DF.
\end{abstract}

Palavras-chave: Exercício. Motivação. Centros de Convivência e Lazer. Avaliação de Programas e Projetos de Saúde.

\section{INTRODUÇÃO}

Com maior ênfase nas duas últimas décadas, as atividades físicas vêm se firmando como um dos marcantes fenômenos sociais do

\footnotetext{
* Mestre em Educação Física. Assessora Técnica da Gerência de Estudos e Pesquisas do Serviço Social do Comércio. Departamento Nacional. Rio de Janeiro, RJ, Brasil. E-mail: mscastro@sesc.com.br

** Mestre em Educação Física. Docente da Universidade Gama Filho. Rio de Janeiro, RJ, Brasil. E-mail: mirandamarcia@uol.com.br

*** Doutora em Educação Física. Docente da Universidade do Estado do Rio de Janeiro. Rio de Janeiro, RJ, Brasil. E-mail: nadialima@globo.com

**** Doutor em Saúde Pública. Docente da Universidade Federal do Rio de Janeiro, Rio de Janeiro, RJ, Brasil. E-mail: palma_alexandre@yahoo.com.br

***** Livre-Docente em Educação Física. Vice-Reitor de Pós-Graduação, Pesquisa e Extensão da Universidade Castelo Branco. Rio de Janeiro, RJ, Brasil. E-mail: heldergr@globo.com
} 
planeta (RUBIO, 2002). Esse fenômeno parece traduzir a consolidação de valores que permeiam a prática de exercícios físicos, desde os tempos da Antiguidade (LOVISOLO, 2002; RUBIO, 2002; SALLES-COSTA, 2003). Estes valores se firmaram em conformidade com o contexto histórico e cultural das sociedades, e vêm se revezando em ordem de prioridade, influenciando ideais como a modelação estética, a prevenção de doenças, a aptidão física e a manutenção da saúde.

Nos últimos anos, muitos dos estudos que destacam a importância da prática de exercício físico no tempo livre e, consequentemente, os motivos que levam a adesão de um comportamento fisicamente ativo, são motivados pela associação temática com a saúde na perspectiva biomédica. Pautadas nessa perspectiva, várias pesquisas foram desenvolvidas defendendo a ideia de que a prática regular de atividades físicas pode contribuir para a prevenção de doenças crônicodegenerativas e para a diminuição da morbimortalidade causada por essas doenças (MATSUDO et al., 2002; PITANGA, 2002).

Algumas pesquisas (SESC/SP, 2003) demonstram que a população está ciente e acreditando nos benefícios da realização de atividade física para a saúde. No entanto, verifica-se um paradoxo entre essa crença e a adoção de um estilo de vida fisicamente ativo (LOVISOLO, 2002). Muito se pergunta por que após tantas campanhas em prol deste tipo de prática: Mexa-se, Agita São Paulo, Sport for All, TRIM, ParticipAction entre outros, os níveis porcentuais de pessoas fisicamente ativas não têm progredido em todo o mundo (POWELL et al., 1991). Em resposta a essa questão, verifica-se um aumento gradativo de pesquisas voltadas para a denúncia acerca da limitação da abordagem biomédica, desenvolvendo, em contraponto, questões em torno de um entendimento da saúde ampliada, na qual se considera o contexto socioeconômico, ambiental e histórico-cultural de indivíduos e populações, que poderiam explicar melhor o fenômeno da adesão ao exercício físico regular (ESPIRITO SANTO; MOURÃO, 2004).

Diante do quadro apresentado, é incontestável a necessidade de ampliar as investigações sobre o tema. Nessa direção, o presente estudo, pautado no referencial da Teoria Explicativa das Condutas Humanas: norma, utilidade e gosto (LOVISOLO, 1995), das Teorias

Movimento, Porto Alegre, v. 15, n. 02, p. 87-102, abril/junho de 2009. 
do Comportamento Planejado e Social Cognitiva e no Modelo Transteórico dos Estágios de Mudança do Comportamento (DISHMAN, 1994), teve por objetivo identificar os motivos que influenciam a adesão dos praticantes de exercícios físicos que frequentam os programas oferecidos em Centros de Atividades do SESC, no Distrito Federal, além de verificar se eles diferenciam conforme sexo, idade e tipos de exercícios físicos.

\section{Metodologia}

\subsection{PopulaÇÃo E AMOSTRA}

A amostra foi selecionada a partir da população de 12.845 sujeitos, com idades a partir de 15 anos, inscritos, em pelo menos, uma modalidade físico-esportiva dos seis Centros de Atividades do SESC/ DF. Foi utilizada a listagem dos alunos inscritos em todas as modalidades físico-esportivas do SESC/DF, sendo eliminadas por sorteio as replicações de alunos inscritos em mais de uma modalidade ou turma, de forma a manter a mesma probabilidade de seleção.

A amostra foi composta de forma aleatória simples, sendo proporcional e representativa dos praticantes de cada Centro de Atividades do SESC/DF (Tabela 1), totalizando 986 sujeitos de ambos os sexos, definidos por sorteio.

Tabela 1. Amostra populacional por Centro de Atividades do SESC/DF

\begin{tabular}{|l|c|c|}
\hline \multicolumn{1}{|c|}{ Unidade SESC } & População & Amostra \\
\hline 504 Sul & 3.208 & 246 \\
\hline 913 Sul & 1.895 & 145 \\
\hline Gama & 1.015 & 79 \\
\hline Guará & 1.762 & 135 \\
\hline Taguatinga Norte & 3.620 & 278 \\
\hline Taguating Sul & 1.345 & 103 \\
\hline Total & $\mathbf{1 2 . 8 4 5}$ & $\mathbf{9 8 6}$ \\
\hline
\end{tabular}

\subsection{ColetA E ANÁLISE DOS DADOS}

Este trabalho seguiu as orientações técnicas do método da pesquisa do tipo survey interseccional (BABBIE, 2001), cuja coleta dos

Movimento, Porto Alegre, v. 15, n. 02, p. 87-102, abril/junho de 2009. 
dados foi realizada no período de 18 a 28 de outubro de 2005 , através de um questionário autoadministrado, construído e validado por Alves et al. (2007). Apesar de autoadministrado, os indivíduos tiveram a oportunidade de esclarecer dúvidas com o profissional responsável pela aplicação do questionário. O mesmo foi respondido no próprio SESC, no horário das aulas.

A primeira parte do instrumento foi composta por perguntas sobre o perfil sociodemográfico da população e a segunda parte apresentou uma questão fechada, cujo objetivo foi identificar os motivos que influenciaram o ingresso dos participantes no programa de exercício físico. Nessa segunda parte, os respondentes puderam indicar até três motivos para o ingresso no(s) programa(s) de exercício(s) físico(s) praticado(s), atribuindo, em ordem de importância, o número 1 para o principal motivo, 2 para o segundo principal motivo e 3 para o terceiro principal motivo. Para as respostas marcadas com o número 1 foram creditados três pontos; respostas com o número 2, dois pontos; e respostas com o número 3, um ponto. $\mathrm{O}$ tratamento dos dados foi realizado por meio do somatório dos pontos registrados em cada item escolhido pelos respondentes e, assim, identificado os que obtiveram maior pontuação. Foram classificados como motivos mais importantes aqueles que, pela escolha dos informantes, obtiveram o valor porcentual igual ou superior a $10 \%$.

Os dados foram analisados por meio de estatística descritiva pelo pacote estatístico SPSS (versão 13).

A caracterização do perfil da amostra e análise dos dados foi realizada considerando os seguintes aspectos: sexo; faixa etária; escolaridade; renda familiar; tipos de exercícios físicos; situação conjugal; e motivos de ingresso no programa de exercício físico.

\section{Resultados E discussão}

A Tabela 2 apresenta uma síntese das características sociodemográficas da amostra estudada, em que são descritos somente os itens que apresentaram maior prevalência e importância para a análise dos dados.

Movimento, Porto Alegre, v. 15, n. 02, p. 87-102, abril/junho de 2009. 
De acordo com Dishman (citado por ANDREOTTI; OKUMA, 2003), os fatores pessoais, mais especificamente os fatores sociodemográficos (Variáveis Determinantes do Comportamento Humano), exercem importante influência sobre o ingresso de indivíduos nos programas de exercícios físicos, sendo mais facilmente encontrados entre os que aderiram à prática, indivíduos de bom nível econômico e de escolaridade, adultos e casados. Os dados encontrados no presente estudo confirmam a argumentação do autor.

Tabela 2. Caracterização sociodemográfica da amostra

\begin{tabular}{|l|c|}
\hline \multicolumn{1}{|c|}{ Variáveis } & Valores percentuais \\
\hline Idade & $44,60 \%$ \\
$21-40$ anos & $28,19 \%$ \\
$41-60$ anos & \\
\hline Sexo & $64,90 \%$ \\
Feminino & $35,10 \%$ \\
Masculino & \\
\hline Renda familiar & $33,00 \%$ \\
Acima de R $\$ 3.000,00$ & $25,30 \%$ \\
Entre R $\$ 1.800,00$ e $\mathrm{R} \$ 3.000,00$ & \\
\hline Escolaridade & $41,40 \%$ \\
Nível superior & $58,60 \%$ \\
Demais níveis & \\
\hline Situação conjugal & $51,90 \%$ \\
União estável & $34,10 \%$ \\
Solteiros & \\
\hline Tipo de atividade & $57,90 \%$ \\
Ginástica & $38,40 \%$ \\
Esportes individuais & \\
\hline
\end{tabular}

Como pode ser visto na Tabela 2, a maior concentração de indivíduos que praticam exercícios físicos no SESC/DF são jovens, mulheres, detentores de boa formação acadêmica, alto poder aquisitivo, que vivem em situação de união estável, e cuja preferência de prática físico-esportiva é a ginástica.

Com relação à faixa etária, a maioria dos indivíduos que pratica exercícios físicos no SESC/DF encontra-se entre 21 e 40 anos de idade, seguida do grupo etário de 41 a 60 anos. Esses resultados demonstram similaridade com outros estudos, a exemplo do realizado por Palma et al. (2003) e Monteiro et al. (2003).

Movimento, Porto Alegre, v. 15, n. 02, p. 87-102, abril/junho de 2009. 
A maior parte do grupo de praticantes de exercícios físicos do SESC/DF é constituída pelas mulheres (64,9\%), resultado que contradiz alguns estudos que demonstram que os homens são mais fisicamente ativos no lazer do que as mulheres (MONTEIRO et al., 2003; SALLES-COSTA et al., 2003; IBGE, 1999). Uma hipótese para esses diferentes resultados pode estar no tipo de exercício físico praticado, já que a ginástica aparece como a maior opção, o que corrobora estudo desenvolvido por Salles-Costa et al. (2003) sobre gênero e prática de exercícios físicos de lazer. Neste estudo, foi constatado que a presença masculina é maior em esportes coletivos como o futebol e o vôlei, assim como na musculação e na corrida, enquanto que, entre as mulheres, a preferência é pela ginástica, hidroginástica, dança e caminhada.

Autores como Palma et al. (2006) e Pate et al. (1995) afirmam que o status econômico tem uma relação direta com a prática de exercícios físicos, o que foi confirmado no presente estudo, pois constatou-se que os participantes dos programas de exercício físico do SESC/DF com renda familiar acima de $\mathrm{R} \$ 3.000,00$ constituem a maioria da amostra.

Quanto à escolaridade, $41,4 \%$ dos indivíduos da amostra do presente estudo possuem nível superior. Esse dado vai ao encontro de outras pesquisas que indicam uma relação direta entre escolaridade e prática de exercícios físicos (NUNES; BARROS, 2004; VIEIRA; FERREIRA, 2004).

Os indivíduos em situação de união estável representam 51,9\% da amostra e os solteiros, $34,1 \%$. Esses dados corroboram os achados da pesquisa de Vieira e Ferreira (2004) e Storchi e Nahas (1992). Estes últimos encontraram que praticantes de atividades físicas espontâneas de Florianópolis são 90,8\% de homens e 78,7\% de mulheres casadas. A suposição dos autores é que esses dados se justificam quando relacionados à faixa etária investigada, caracterizada como de meia idade. Esse fato também pode ser considerado na presente pesquisa, uma vez que houve a predominância de participantes nas faixas etárias entre 21-40 anos de idade (44,6\%) e 41-60 anos (28,19\%).

Nas tabelas que se seguem, apresentam-se os principais motivos alegados pelos indivíduos para ingressarem nos programas de exercícios

Movimento, Porto Alegre, v. 15, n. 02, p. 87-102, abril/junho de 2009. 
físicos (Tabela 3), bem como se esses motivos se diferenciam quando relacionados às variáveis: idade (Tabela 4), sexo (Tabela 5) e tipos de exercícios (Tabela 6). Na Tabela 3, destacam-se apenas os motivos que alcançaram uma pontuação a partir de $10 \%$ de prevalência.

Tabela 3. Principais motivos de ingresso nos programas de exercícios físicos, segundo os indivíduos da amostra

\begin{tabular}{|l|c|c|}
\hline \multicolumn{1}{|c|}{ Motivos } & Pontos & \% \\
\hline Médico aconselhou & 1064 & 17,99 \\
\hline Evita problemas de saúde & 857 & 14,49 \\
\hline Melhora o condicionamento físico & 725 & 12,25 \\
\hline Queria ou precisava emagrecer & 588 & 9,94 \\
\hline Queria me sentir bem físico e emocionalmente & 495 & 8,37 \\
\hline Sempre gostou de fazer atividade(s) físico-esportiva(s) & 463 & 7,83 \\
\hline Sente prazer em fazer exercícios & 459 & 7,76 \\
\hline Queria ter um corpo mais bonito & 398 & 6,73 \\
\hline Facilidade de acesso & 265 & 4,48 \\
\hline Estar com amigos e fazer novas amizades & 124 & 2,10 \\
\hline Aprender/praticar nova(s) atividade(s) físico-esportiva(s) & 112 & 1,89 \\
\hline Mensalidade mais adequada às possibilidades de pagamento & 90 & 1,52 \\
\hline Queria ser um(a) atleta & 68 & 1,15 \\
\hline Responsáveis decidiram pela prática de atividade física & 67 & 1,13 \\
\hline Não respondeu & 46 & 0,78 \\
\hline Viu muitas propagandas, reportagens e programas & 40 & 0,68 \\
\hline Possibilitam diferentes desafios & 37 & 0,63 \\
\hline Pessoas que praticam são mais valorizadas & 18 & 0,30 \\
\hline Total & $\mathbf{5 9 1 6}$ & $\mathbf{1 0 0}$ \\
\hline
\end{tabular}

Tabela 4. Principais motivos de ingresso nos programas de exercícios físicos, segundo a faixa etária

\begin{tabular}{|l|c|c|}
\hline \multicolumn{1}{|c|}{ Motivos } & $\mathbf{n}$ & $\%$ \\
\hline Faixa etária: 15 a 20 anos & 103 & 16,19 \\
Queria ter um corpo mais bonito & 87 & 13,68 \\
Sempre gostou de fazer atividade(s) físico-esportiva(s) & 67 & 10,53 \\
Melhora o condicionamento físico & & \\
\hline Faixa etária: 21 a 40 anos & 386 & 14,64 \\
Melhora o condicionamento físico & 343 & 13,01 \\
Evita problemas de saúde & 316 & 11,98 \\
Queria ou precisava emagrecer & 303 & 11,49 \\
Médico aconselhou & & \\
\hline
\end{tabular}

continua...

Wovimento, Porto Alegre, v. 15, n. 02, p. 87-102, abril/junho de 2009. 


...continuação
\begin{tabular}{|l|r|l|}
\hline Faixa etária: 41 a 60 anos & 401 & 24,04 \\
Médico aconselhou & 292 & 17,51 \\
Evita problemas de saúde & 192 & 11,51 \\
Melhora o condicionamento físico & & \\
\hline Faixa etária: $>60$ anos & 314 & 32,21 \\
Médico aconselhou & 184 & 18,87 \\
\hline Evita problemas de saúde &
\end{tabular}

Tabela 5. Principais motivos de ingresso nos programas de exercícios físicos,

\begin{tabular}{|l|c|c|}
\multicolumn{2}{|c|}{ Megundo o sexo } \\
\hline \multicolumn{1}{|c|}{ Motivos } & $\mathbf{n}$ & $\%$ \\
\hline Sexo Feminino & & \\
Médico aconselhou & 794 & 20,68 \\
Evita problemas de saúde & 597 & 15,55 \\
Melhora o condicionamento físico & 443 & 11,54 \\
Queria ou precisava emagrecer & 436 & 11,35 \\
\hline Sexo Masculino & & \\
Melhora o condicionamento físico & 282 & 13,58 \\
Médico aconselhou & 270 & 13,01 \\
Evita problemas de saúde & 260 & 12,52 \\
Sempre gostou de fazer atividade(s) físico-esportiva(s) & 220 & 10,60 \\
\hline
\end{tabular}

Tabela 6. Principais motivos de ingresso nos programas de exercícios físicos, segundo o(s) tipo(s) de exercício(s) físico(s)

\begin{tabular}{|l|c|c|}
\hline \multicolumn{1}{|c|}{ Motivos } & n & $\%$ \\
\hline Esporte individuais & 434 & 19,11 \\
Médico aconselhou & 323 & 14,22 \\
Melhora o condicionamento físico & 297 & 13,08 \\
Evita problemas de saúde & & \\
\hline Esportes coletivos & 108 & 25,71 \\
Médico aconselhou & 50 & 11,90 \\
Evita problemas de saúde & & \\
\hline Ginástica & 543 & 15,88 \\
Médico aconselhou & 532 & 15,56 \\
Evita problemas de saúde & 394 & 11,52 \\
Melhora o condicionamento físico & 390 & 11,40 \\
Queria ou precisava emagrecer & 342 & 10,00 \\
Queria me sentir bem físico e emocionalmente & & \\
\hline Lutas & 11 & 18,33 \\
Queria ter um corpo mais bonito & 10 & 16,67 \\
Sempre gostou de fazer atividade(s) físico-esportiva(s) & 8 & 13,33 \\
Sente prazer em fazer exercícios & 8 & 13,33 \\
Queria ou precisava emagrecer & 7 & 11,67 \\
Evita problemas de saúde & 6 & 10,00 \\
Melhora o condicionamento físico &
\end{tabular}

Movimento, Porto Alegre, v. 15, n. 02, p. 87-102, abril/junho de 2009. 
Considerando os resultados apresentados sobre os motivos que justificam o ingresso do grupo de indivíduos nos programas de exercícios físicos do SESC/DF, verifica-se pouca variação dos propósitos indicados quando se compara o resultado geral apresentado na Tabela 3 com os resultados parciais obtidos a partir da análise das variáveis: idade (Tabela 4), sexo (Tabela 5) e tipos de exercícios (Tabela 6).

Os itens relacionados à saúde e ao condicionamento físico foram os mais indicados na maioria das variáveis analisadas.

Cabe destacar a possibilidade de que os sujeitos da pesquisa tenham se valido de uma "resposta politicamente correta", já que a proteção da saúde constitui-se em uma espécie de marca discursiva recorrente entre os especialistas da área da saúde e nos meios de comunicação de massa. Portanto, trata-se de um motivo bastante disseminado na sociedade.

Em um discurso de coerência conceptual, a busca da potencialização e da manutenção das capacidades físicas (força e resistência muscular, condicionamento cardiorrespiratório, flexibilidade e composição corporal), representado neste estudo pela opção "Melhora o condicionamento físico", é defendido e difundido pelos especialistas identificados com o movimento pelo estilo de vida fisicamente ativo, como um motivo que se articula com a proteção da saúde (NAHAS, 2001). O conceito de aptidão física relacionado à saúde vem sendo disseminado desde a década de 1970, a partir dos protocolos formulados pela Aliança Americana para a Saúde, Educação Física, Recreação e Dança como condição essencial de aptidão para a vida. Para esta concepção, o trabalho das capacidades físicas é fundamental para caracterizar um estilo de vida ativa, com menos riscos de contração de doenças hipocinéticas e maior perspectiva de uma vida mais longa e autônoma (NAHAS, 2001). Deste modo, era de se esperar que este motivo aparecesse com uma incidência similar aos relacionados à saúde biomédica.

Os motivos relacionados à estética e ao prazer também aparecem entre os mais indicados, porém, com menos força que os três primeiros. Contudo, em conversas desprovidas de formalidades, nos quais é menor o apelo em prol de depoimentos "politicamente corretos", é

Movimento, Porto Alegre, v. 15, n. 02, p. 87-102, abril/junho de 2009. 
frequente a associação entre o início da prática de exercícios físicos e o propósito da busca de um padrão estético idealizado. Alguns estudos que utilizaram como metodologia a análise de conteúdo ou de discurso revelam que este desejo tem grande força na decisão de indivíduos iniciarem práticas de exercícios físicos (PEREIRA, 1996).

Um dado que pode ter influenciado a diferença entre os motivos voltados para a saúde apresentados neste estudo como mais fortes e o apontado por Pereira (1996), é o fato de a amostra ser constituída em sua maioria por sujeitos adultos jovens e de meia idade (Tabela 2), cuja maturidade pode representar uma tendência de maior cuidado com aspectos relacionados à saúde em detrimento dos relacionados à estética corporal, motivo que mais apareceu na faixa etária dos adolescentes (Tabela 4).

Em que pese tais reflexões, recorrendo ao referencial teórico que serve de apoio à análise dos resultados deste estudo, pode-se inferir que, com base na Teoria Explicativa das Condutas Humanas (LOVISOLO, 1995), os indivíduos foram influenciados a ingressar nos programas de exercícios físicos por mais de um motivo, geralmente os relacionados à norma e à utilidade (aconselhamento médico, prevenção de problemas de saúde e melhora do condicionamento físico), embora também tenha sido encontrada a presença concomitante dos três motivos (norma, utilidade e gosto), no entanto, em menor quantidade de variáveis. Esse resultado confirma que apenas um dos motivos, independentemente dos outros dois, não é capaz de influenciar um determinado comportamento, quando se trata do universo de possibilidades de acesso à prática de exercícios físicos.

De acordo com a Teoria do Comportamento Planejado (DISHMAN, 1994) e com as Variáveis Determinantes do Comportamento, no que concerne aos fatores pessoais (DISHMAN citado por ANDREOTTI; OKUMA, 2003), os resultados apontam que a amostra agiu, preponderantemente, influenciada pela crença e pela norma social, que credita à prática de exercícios físicos o alcance dos benefícios para a saúde. Este resultado sugere a crença na dimensão biomédica da saúde de que condutas individuais, regradas e fisicamente ativas, são os fatores preponderantes de promoção da saúde. Esta

Movimento, Porto Alegre, v. 15, n. 02, p. 87-102, abril/junho de 2009. 
perspectiva parece ser a mais forte justificativa para o ingresso de jovens, adultos e idosos nos programas de exercícios físicos oferecidos pelo SESC/DF, independentemente de serem homens ou mulheres.

Recorrendo aos que apregoam a Teoria do Comportamento Planejado e o Modelo Transteórico dos Estágios de Mudança do Comportamento (DISHMAN, 1994), os resultados sugerem que pode estar havendo efetividade das campanhas de sensibilização, entre outras estratégias que influenciam crenças e os comportamentos em prol da adesão à prática de exercícios físicos, na perspectiva da relação saúde-prevenção de doenças. O Modelo Transteórico dos Estágios de Mudança do Comportamento defende que, no estágio de pré-contemplação, a estratégia indicada é enfatizar os benefícios que poderão ser alcançados por meio dos hábitos recomendados para que os indivíduos possam ser estimulados a iniciar a prática. Ações de sensibilização com base na crença dos benefícios da ação também são recomendadas pela Teoria do Comportamento Planejado. Essas hipóteses têm sido reforçadas por meio da bibliografia.

Andreotti e Okuma (2003), por exemplo, citam que, para Dishman, a recomendação médica para a prática de exercícios físicos estimula consideravelmente o ingresso nos programas, principalmente para os idosos. Vieira e Ferreira (2004) também admitem que possa haver uma tendência para que a associação entre aptidão física e saúde esteja contribuindo para a adesão à prática de exercícios físicos. Caminhando nesse sentido, percebe-se que os motivos relacionados à saúde são apontados na maior parte das pesquisas sobre o tema. No entanto, nem sempre a saúde é confirmada como o fator mais importante.

Em pesquisa realizada por Vieira e Ferreira (2004) sobre os praticantes de atividades físicas em torno do estádio de futebol do Maracanã, no Rio de Janeiro, verificou-se que os principais motivos que levaram os informantes a praticar exercícios foram a saúde $(72,3 \%)$, o emagrecimento $(42,9 \%)$ e a recomendação médica $(35,9 \%)$.

Andreotti e Okuma (2003), ao realizarem pesquisa sobre o perfil sócio-demográfico e de adesão inicial de idosos ingressantes em um programa de Educação Física, constataram que o principal motivo de ingresso foi a indicação de amigos, destacado por 17 indivíduos

Movimento, Porto Alegre, v. 15, n. 02, p. 87-102, abril/junho de 2009. 
entre os 44 pesquisados. O motivo "melhorar a saúde" apareceu como o segundo principal, indicado por 13 sujeitos.

Alves (2004), em pesquisa sobre os motivos que influenciam os adolescentes a ingressar e a permanecer praticando natação, encontrou como principais motivos de ingresso os seguintes itens: "porque eu sempre gostei de fazer natação" (16,04\%), "porque meus pais ou responsáveis decidiram que era importante eu praticar natação" (14,95\%), "porque o médico me aconselhou" $(10,45 \%)$ e "porque eu não sabia nadar" $(10,27 \%)$. O item que aponta o aconselhamento médico parece demonstrar a preocupação com a saúde, relacionada com questões ligadas ao prazer, ao aprendizado da natação e à influência dos responsáveis.

Os dados levantados na presente pesquisa corroboram os achados de Vieira e Ferreira (2004), na medida em que chamam a atenção para a predominância dos motivos relacionados à saúde/prevenção de doenças (classificados como os principais na maior parte das variáveis) como os de maior influência na decisão dos indivíduos ingressarem nos programas de exercícios físicos do SESC/DF.

\section{CONSIDERAÇÕES FINAIS}

Considerando o objetivo do presente estudo, bem como os resultados que ilustram os motivos de adesão dos usuários de programas de exercícios físicos do SESC/DF, constatou-se a presença da crença e dos interesses relacionados aos benefícios desse tipo de atividade para saúde biomédica, como um forte indicador da prática de exercícios físicos. Nos motivos de ingresso, percebe-se claramente o viés da saúde, por intermédio do aconselhamento médico e do desejo de evitar doenças, como os principais motivos apontados pelos informantes.

Portanto, como demonstrado pelos dados analisados e pela bibliografia utilizada, insistir no principal argumento da saúde biomédica pode contribuir para resultados pertinentes à adesão inicial (ingresso) dos indivíduos em programas de exercícios físicos. Todavia, em que pese não ter sido objeto de análise deste estudo, cabe aqui ressaltar

Movimento, Porto Alegre, v. 15, n. 02, p. 87-102, abril/junho de 2009. 
que Castro (2006) levantou dados que sugerem que esse argumento não é suficiente para a manutenção da prática.

Outro dado posto pela bibliografia, e verificado nos resultados desta pesquisa, diz respeito à prevalência do perfil de praticantes de nível socioeconômico privilegiado. O que parece é que muitas intervenções, mesmo que apresentem o objetivo de acessibilidade e democratização da prática, não conseguem envolver e atrair a parcela da população menos favorecida, independentemente dos seus possíveis interesses (gosto, utilidade e/ou norma).

Tornar essas práticas acessíveis à população dos mais variados níveis socioeconômicos apresenta-se como um desafio. Mas, para esse fim, é necessário reconhecer que para a parcela da população menos favorecida e mais vulnerável, existem barreiras impostas também pelas más condições de vida, determinadas pelas desigualdades sociais (PALMA et al., 2006; PALMA, 2000).

Como defendido por alguns autores (PALMA, 2000), saúde é a resultante das condições de vida de indivíduos e coletividades, traduzidas em renda, moradia, serviços de atenção à saúde, lazer, educação, dentre outros. Nesse sentido, a função das políticas públicas e dos programas de intervenção deve ser a de assegurar a devida formação cultural e garantir os meios necessários para que as pessoas possam ter acesso a esse acervo cultural, compreendido como direito inalienável de todo cidadão e como importante patrimônio histórico da humanidade e do processo de construção da individualidade humana.

Movimento, Porto Alegre, v. 15, n. 02, p. 87-102, abril/junho de 2009. 

Reasons of Ingression in the Programs of Physical
Exercises Offered by the Social Service of the Commerce-SESC-DF

Abstract: The objective of this study was to identify the reasons of adherence of the practitioners of physical exercises of the Centers of Activities of the SESC-DF and to verify if they differentiate according to sex, age and types of physical exercises. A representative sample of 986 individuals of both sexes (15 years or more) answered a questionnaire constructed and validated by Alves et al. (2007). The data reveal that the informants entered the programs (a) following medical indication, (b) to prevent problems of health and (c) to improve the physical conditioning. These results suggest that the argument concerning health is the main reason that justifies the adherence to the programs of physical exer-cises of the SESC-DF.

Keywords: Exercise. Motivation. Centers of Connivance and Leisure. Program Evaluation.

\footnotetext{
Las Razones de Ingreso en los Programas de Ejercicios Físicos Ofrecidos por el Servicio Social del Comércio-SESC-DF

Resumen: El objetivo de este estudio era identificar las razones del ingreso de las personas que hacen actividades físicas en los centros de las actividades del SESC-DF y verificar si ellas si distinguen según sexo, edad y los tipos de ejercicios físicos. Una muestra representativa de 986 individuos de ambos los sexos (15 años o más) contestó a un cuestionario construido y validado por Alves et al. (2007), cuyos dados divulgan que las personas habían incorporado los programas (a) por indicación médica, (b) para prevenir problemas de la salud y (c) para mejorar el condicionamiento físico. Esos resultados sugieren que la discusión de la salud sea la razón principal que justifica la adherencia a los programas de los ejercicios físicos del SESC-DF.

Palabras Clave: Ejercicio. Motivación. Centros de Ocio y Convivência. Evaluación de Programas y Proyectos de Salud.
}

\section{REFERÊNCIAS}

ALVES, M. P. Estudo dos motivos que influenciam os adolescentes a ingressar e permanecer praticando natação. 96 f. Dissertação (Mestrado) Programa de Pós-Graduação Stricto Sensu em Educação Física, Universidade Gama Filho. Rio de Janeiro, 2004.

Movimento, Porto Alegre, v. 15, n. 02, p. 87-102, abril/junho de 2009. 
ALVES, M. P. et al. Motivos que justificam a adesão de adolescentes à prática da natação: qual o espaço ocupado pela saúde? Revista Brasileira de Medicina do Esporte, Rio de Janeiro, v. 13, n. 6, p. 421-426, 2007.

ANDREOTTI, M. C.; OKUMA, S. S. Perfil sociodemográfico e de adesão inicial de idosos ingressantes em um programa de educação física. Revista Paulista de Educação Física, São Paulo, v. 17, n. 2, p.142-153, 2003.

BABBIE, E. Métodos de pesquisa de survey. Belo Horizonte: UFMG, 2001.

CASTRO, M. S. Motivos que influenciam a adesão à prática de exercícios físicos, nos programas oferecidos pelo Serviço Social do Comércio (SESC), no Distrito Federal. 127 f. Dissertação (Mestrado). - Programa de PósGraduação Stricto Sensu em Educação Física, Universidade Gama Filho. Rio de Janeiro, 2006.

DISHMAN, R. K. The measurement conundrum in exercise adherence research. Medicine and Science in Sports and Exercise, Indianapolis, v. 26, n. 11, p. 13821390, 1994.

ESPIRITO SANTO, Giannina; MOURÃO, Ludmila. Um debate sobre a trajetória da produção do conhecimento em atividade física e saúde nos 25 anos de CBCE. Motus Corporis, Rio do Janeiro, v. 11, n. 1, p. 29-44, 2004.

INSTITUTO BRASILEIRO DE GEOGRAFIAE ESTATÍSTICA. Pesquisa sobre padrões de vida 1996-1997. Rio de Janeiro, IBGE, 1999.

LOVISOLO, H. Atividade física e saúde: uma agenda sociológica de pesquisa. In: MOREIRA, W. W.; SIMÕES R. (Org.). Esporte como fator de qualidade de vida. Piracicaba: UNIMEP, 2002. p. 277-298.

LOVISOLO, H. Normas, utilidades e gostos na aprendizagem. In: VOTRE Sebastião Josué, COSTA, V. L. M. (Org.). Cultura, atividade corporal e esporte. Rio de Janeiro: Editoria Central da Universidade Gama Filho, 1995. p. 213-31.

MATSUDO, S. M. et al. Nível de atividade física da população do estado de São Paulo: análise de acordo com gênero, idade, nível socioeconômico, distribuição geográfica e conhecimento. Revista Brasileira de Ciência e Movimento, Brasília, v. 10, n. 4, p. 41-50, 2002.

MONTEIRO, C. A. et al. A descriptive epidemiology of leisure-time physical activity in Brazil, 1996-1997. Revista Panamericana Salud Publica, Washington, v. 14, n. 4, p. 246-254, 2003.

NAHAS, M. V. Conceitos e sugestões para um estilo de vida ativo. Londrina: Midiograf, 2001.

NUNES, J. O. M.; BARROS, J. F. Fatores de risco associados à prevalência de sedentarismo em trabalhadores da indústria e da Universidade de Brasília. Efdeportes: revista digital Buenos Aires. v. 10, n. 69, 2004. Disponível em: <http:/ /www.efdeportes.com.> Acesso em: 17de junho de 2005.

Movimento, Porto Alegre, v. 15, n. 02, p. 87-102, abril/junho de 2009. 
PALMA, A. Atividade física, processo saúde-doença e condições sócio-econômicas: uma revisão de literatura. Revista Paulista de Educação Física, São Paulo, v. 14 n. 1 , p. $97-106,2000$.

PALMA, A. et al. Reflexões acerca da adesão aos exercícios físicos: comportamento de risco ou vulnerabilidade? Movimento, Porto Alegre, v. 9, n. 3, p. 83-100, 2003.

PALMA, A. et al. Dimensões epidemiológicas associativas entre indicadores sócioeconômicos de vida e prática de exercícios físicos. Revista Brasileira de Ciências do Esporte, Campinas, v. 27, n. 3, p. 119-136, 2006.

PATE, R. et al. Physical activity and public health. A recommendation from the Centers for Disease Control and Prevention and the American College of Sports Medicine. JAMA, Columbia, v. 273, n. 5, p. 402-407, 1995.

PEREIRA, G. B. P. Ginástica de academia: potência de ser e equilíbrio pessoal. 126 f. Dissertação (Mestrado) - Programa de Pós-Graduação Stricto Sensu em Educação Física, Universidade Gama Filho. Rio de Janeiro, 1996.

PITANGA, Francisco José G. Epidemiologia, atividade física e saúde. Revista Brasileira de Ciência e Movimento, Brasília, v. 10, n. 3, p. 49-54, 2002.

POWELL, K. E. et al. Progress and problems in the promotion of physical activity. In OJA P.; TELAMA, R. (orgs). Sport for all, Amsterdan: Elsevier Science, 1991. p. 55 -73 .

RUBIO, K. Do Olimpo ao pós-olimpismo: elementos para reflexão do esporte atual. Revista Paulista de Educação Física, São Paulo, v. 16, n. 2, p. 130-143, 2002.

SALLES-COSTA, R. et al. Gênero e prática de atividade física no lazer. Cadernos de Saúde Pública, Rio de Janeiro, v. 19, n. 2, p. S325 - S333, 2003.

SERVIÇO SOCIAL DO COMÉRCIO. Escolhas sobre o corpo: valores e práticas em tempo de mudança. São Paulo: SESC/SP, 2003.

STORCHI, C. M.; NAHAS, M. V. A Prática espontânea de atividades físicas nas ruas de Florianópolis, SC: diagnóstico preliminar com indivíduos de meia idade. Revista Brasileira de Ciências do Movimento, Brasília, v. 6, n. 1, p. 7-13, 1992

VIEIRA, V.; FERREIRA, M. S. Perfil de praticantes de atividade física na pista do Maracanã. Revista Ação e Movimento, São Paulo, v. 1, n. 2, p. 81-90, 2004.

Recebido em: 24.10.2007

Aprovado em: 17.06.2008

Movimento, Porto Alegre, v. 15, n. 02, p. 87-102, abril/junho de 2009. 